\title{
LA HERMENÉUTICA Y LOS TEXTOS: EL RETO DE LA TRADUCCIÓN
}

HERMENEUTICS AND TEXTS: THE CHALLENGE OF TRANSLATION

Teresa Jesús Ríos Delgado ${ }^{*}$

triosd@unmsm.edu.pe

${ }^{1}$ Universidad Nacional Mayor de San Marcos, Lima, Perú

*Correspondencia: Teresa Jesús Ríos Delgado. Email: triosd@unmsm.edu.pe

Recibido: 15.07.21 | Aprobado: 14.08.21

\section{RESUMEN}

El presente artículo presenta el análisis de la hermenéutica como traducción, relacionando ambos conceptos en el desarrollo de diferentes visiones de la realidad, del mundo, y sus acciones del ser humano en su afán de justificar básicamente su comportamiento o el camino en su vida. Asimismo, se aprecia la perspectiva hermenéutica de la traducción en base a autores como Wittgenstein, Ricoeur y Gadamer, donde el texto es el eje central de la comprensión e interpretación. El traductor no solo es el puente entre dos mundos, sino también es el intérprete de la realidad en ambas lenguas y es el responsable de una interpretación que plasme la comprensión y aceptación de estas realidades. Por último, se presentan, a nuestro criterio, los principales retos de la traducción en la actividad de la interpretación complementándose con ejemplos en los tipos de textos a traducir. La hermenéutica nos permite ver los signos más allá de solo indicadores o al lenguaje como un simple medio de expresión, más bien, apuesta por una relación biunívoca entre el ser humano y el lenguaje.

Palabras claves: hermenéutica, interpretación, traducción

\begin{abstract}
The research work analyzes hermeneutics as translation, relating both concepts, focusing on human being' different visions of reality, world, and actions to basically justify its behavior or way of life. Likewise, the hermeneutical perspective of translation is established based on authors such as Wittgenstein, Ricoeur and Gadamer, where the text is the core of comprehension and interpretation. The translator is not only the bridge between two worlds, but is also the interpreter of reality in both languages and is responsible for an interpretation that reflects the understanding and acceptance of these realities. Finally, in our opinion, the main challenges of translation in the interpreting activity are presented, enhanced with examples of the types of texts to be translated. Hermeneutics allows us to see signs beyond just indicators or language as a simple means of expression, rather, it challenges a one-to-one relationship between human beings and language.
\end{abstract}

Keywords: hermeneutics, interpretation, translation

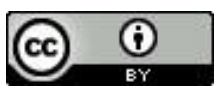




\section{INTRODUCCIÓN}

El mundo se construye desde el "yo" hacia afuera y los fenómenos existen independientemente de nuestra propia construcción. Cada experiencia no puede ser transferida "totalmente como tal" a otro. Esta deseada circularidad es virtuosa, ya que estimula la reflexión hacia una segunda ingenuidad de la compresión e interpretación, ya que comprender, para un ser finito, como el ser humano, es como ser transportado a otra vida, otro mundo o realidad. Estos escenarios también se visualizan en las diferentes lenguas en el mundo, donde la traducción nace con la necesidad del entendimiento, lo cual es parte consustancial de nuestra historia. El texto considerado como objeto en la teoría de la interpretación, o hermenéutica, es también el núcleo de trabajo de la traducción, escenario donde se distingue la traslación de un texto desde la lengua origen (LO) a la lengua meta (LM), como un proceso escrito y la interpretación que se desarrolla en la traslación oral, a través de la comunicación oral de la LO a la LM, es decir texto hablado. Este contexto nos conlleva a desarrollar la relación de la hermenéutica y los textos, principalmente desde la perspectiva de Wittgenstein, Ricoeur y Gadamer, y la traducción, complementando con ejemplos que visualicen los retos de la traducción.

\section{LA HERMENÉUTICA Y LA TRADUCCIÓN}

La hermenéutica y la traducción además de tener un núcleo en común, el texto, nos permiten observar nuestra socialización, pero sobre todo nuestra experiencia frente a la realidad. Contemplamos en Grondin (1999) "entender un texto del pasado significa traducirlo a nuestra situación y escuchar en él una respuesta elocuente a las preguntas de nuestra época” (p. 168), la relación entre hermenéutica y traducción que vamos a desarrollar en las siguientes líneas, en base a la concepción básica de la traducción y la perspectiva de Wittgenstein, Ricoeur y Gadamer.

La traducción, en un sentido general, puede calificarse como una actividad de comunicación intercultural (Sang, 2018), y la hermenéutica se definió como la teoría de la interpretación, inicialmente considerando solo textos. En el siglo XX, los filósofos hermenéuticos, por otro lado, afirman que las experiencias de los escritores y lectores, su experiencia de "vivir en el mundo", su creencia, el contexto de la escritura y la lectura tienen gran importancia para el texto comprensión y, en general, de pensamiento e inteligencia (Trăuşan-Matu, 2017).

Desde nuestra experiencia, creemos que la hermenéutica en el contexto de la traducción es el andamiaje del análisis y comprensión de la realidad, el sentido de los textos y de las palabras despertando esa vena crítica, que todos poseemos, para revelar la realidad y el mundo desde lo racional y filosófico.

Consideramos que la hermenéutica como traducción se debe enfocar no solo en la comprensión del texto para poder traducirlo sino también en el significado de la interpretación y la compresión en el proceso de la traducción. Se está construyendo puentes entre culturas, lo que implica puentes entre diferentes observaciones de la realidad, por cual, podemos decir que el traductor se convierte en una etapa, dentro del proceso de traducción, en un investigador que debe realizar su estado del arte, ya que requiere de analizar y evaluar el mensaje para decodificarlo y trasladarlo a la LM, teniendo 
en cuenta el momento histórico, pero sobre todo las características de la LO en el que fue codificado. La comprensión de textos se constituye en el pilar del traductor.

Desde y para la traducción, es imprescindible además del conocimiento de la lengua, el de su cultura, de su historia, de su sentimiento, efectivamente un espíritu que fue forjado con el acontecer de su uso, cambio y olvido por un determinado grupo social. Entonces, el autor solo puede estar tranquilo si el lector lo está: se cumplió el objetivo de la traducción.

Spoturno (2019) nos habla del ethos del traductor que lo concibe como la imagen discursiva que se asocia a la entidad textual (traductor) y conduce y regula el texto traducido. El ethos del traductor permite mostrar la subjetividad que se vincula con el responsable de la enunciación en el discurso traducido. Para la autora surge en dos niveles: el nivel discursivo, que atañe al posicionamiento, actividad y el compromiso enunciativo que caracterizan la labor del traductor en el texto traducido, los cuales envuelven al traductor en una red tanto sintáctica y semántica como también filosófica, porque el traductor debe estar potencialmente conscientes de los problemas concretos de traducción, que pueda originarse una interpretación en un plano metafísico y que sus preocupaciones son ontológicas más que epistémicas.

"La hermenéutica como traducción, permite apreciar la complejidad y, a la vez, especificidad de la hermenéutica como teoría y como método interpretativo para la comprensión de textos" (Palmer, 1969; citado por Quintana y Hermida, 2019, p. 76). La hermenéutica nos permite ver los signos más allá de como meros indicadores o al lenguaje como un simple medio de expresión, más bien, apuesta por una relación biunívoca entre el ser humano y el lenguaje, posicionando al ser humano como instrumento del lenguaje en lugar del lenguaje como su herramienta.

Consideramos que la traducción tiene un profundo significado filosófico, el brindar valor al sentido siempre abrirá una nueva área de discusión filosófica, puesto que se estimula la reflexión frente al texto a traducir con base en la responsabilidad de que la traslación es comunicación también conocimiento y hasta una verdad. Los métodos que se utilizan para traducir e interpretar son fenómenos lingüísticamente básicos pero que exigen no solo el conocimiento del idioma tanto en LO y LM sino también de las culturas, de la historia de la sociedad que lo utiliza, pero sobre todo del contexto en que se debe traducir.

\section{WITTGENSTEIN Y LOS JUEGOS DEL LENGUAJE}

La hermenéutica considera los textos como medios para transmitir experiencias, creencias y juicios de un sujeto o comunidad a otra. Si tomamos, por ejemplo, la propuesta del segundo Wittgenstein (1988), como los juegos del lenguaje: criterio, semejanzas de familia, forma de vida, estamos en la búsqueda de la compresión de la LO para poder lograr la interpretación en la LM e inmerso en esa búsqueda de la compresión, por lo cual, el uso de la palabra o texto se regirá por el significado que determine el contexto en el cual está siendo usado o también podría ser en base al comportamiento de la persona o sociedad que le dé uso. De esta manera coincidimos con el autor, en relación a que el uso de la palabra depende del contexto en el cual está siendo utilizado, lo que conlleva a 
diversas formas en uso, al juego del lenguaje, que a pesar de tener una delimitación y significado siempre es el vehículo de la comunicación.

¿Es la traducción un juego del lenguaje? ¿El objetivo de la traducción es deshacer los nudos de confusión que el ser humano había venido construyendo a lo largo de su vida? En cierto modo, traducir es un juego del lenguaje, pues existen tantas lenguas como culturas, por lo cual cada lengua se enlaza con una cultura, con su estructura, con su forma de vida. Esa lógica que se utiliza en la construcción, por ejemplo, desde una palabra, hasta construcciones más complejas, como el lenguaje técnico-científico, o sin ir muy lejos en el lenguaje ordinario, como la jerga o el argot, que en algunas ocasiones si es difícil de entender el concepto del término creado, debido a las metáforas a veces descontextualizadas por el escenario en el cual fueron creados. Con tanta pluralidad de interpretaciones, se presenta la oportunidad de seguir conociendo el mundo de diferentes maneras.

Podemos apreciar que Wittgenstein considera al lenguaje como una forma de vida, en el cual los "juegos" se interrelacionan a través de la práctica. La traducción es una actividad y como procedimiento comparte reglas de su proceso, pero al igual que el lenguaje no todo el juego está sometido a normas. Hay subjetividad, emociones, necesidades en el traductor, mientras se inserta en el proceso de identificación y análisis del texto en LO, puede utilizar sus competencias innatas y profesional.

\section{RICOEUR Y LA TRADUCCIÓN}

Por otro lado, el significado de una palabra, por ejemplo, deriva de su oposición a las otras unidades léxicas del mismo tipo. Como dijo Saussure, en un sistema de signos sólo hay diferencias, pero no existencia sustancial" (Ricoeur, 2006, p. 19). Consideramos que la existencia sustancial se traduce a la universalidad que contempla el ser humano día a día, y efectivamente para entender el significado de una palabra, es necesario conocimientos o competencias en el contexto de uso del lenguaje. Un sistema de signos se comprende a través de relaciones sistemáticas con otras expresiones del lenguaje determinado por reglas y definiciones explícitas del propio lenguaje.

Ricoeur (2006) al referirse a la traducción, presenta la temática de la universalidad, la cual considera que se aleja de la temporalidad del lenguaje que se relaciona con la traducción. El significado de las palabras es convención de la sociedad, no de la relación significado / significante, lo que fundamenta el hecho que exista una diversidad de idiomas y seguirá existiendo, pero siguen siendo lenguajes, entonces, esta universalidad del lenguaje contempla la naturaleza de cada una de ellas, es decir Ricoeur, lo establece como una competencia universal. Y coincidimos con Ricoeur en relación a la universalidad, ya que el ser humano ha buscado y sigue buscando un lenguaje universal, y en el camino ha puesto de manifiesto esa gran competencia en la creación de las diversas lenguas. En relación a la traducción, el objetivo es la universalidad de comprensión o entendimiento, pero no total, es utópico pensar que pueda ser total, preferimos usar el término "adecuado". La traducción, tomando como base el proceso de comunicación, es el medio primordial para la comunicación eficaz y efectiva. El texto en LO y LM son productos derivados de diferentes culturas, por lo cual, el patrón lingüístico plasma realidades diferentes en ambos casos, lo que conlleva a una desestabilización en el trabajo de la 
significación. No existe la traducción perfecta, a menos que exista una correspondencia entre los diferentes idiomas: carro - car - wagen (español, inglés, alemán). Sin embargo, si se puede replicar un significado original, ahí radica el éxito de la traducción.

El texto traducido tiene el alma hermenéutica del texto original, porque además de ser su reflejo también está creando un nuevo contexto de cultura, moviéndose entre el tiempo y espacio de esa cultura, articulando una interpretación de las intenciones del texto más que de su equivalencia. El traductor desarrolla una matriz sistémica, donde su "racionalidad humana propende al final, a una concepción de la realidad en la que unos fines determinados actúan como causas explicativas de esa misma realidad, de esta forma la consciencia puede interpretarla como una cadena de causas y efectos" (Tutone, 2019, p. 369).

\section{GADAMER Y TRADUCTOR}

Para Gadamer (1997), el traductor tiene que trasladar el sentido del contexto en el que está el interlocutor (LM), lo que tiene que mantenerse es el sentido, y valerse de la comprensión de ese nuevo mundo lingüístico nuevo: "Toda traducción es por eso ya una interpretación, e incluso puede decirse que es la consumación de la interpretación que el traductor hace madurar en la palabra que se le ofrece" (p. 236). Luego prosigue el autor "cuando es necesaria la traducción no hay más remedio que hacerse cargo de la distancia entre el espíritu de la literalidad originaria de lo dicho y el de su reproducción, distancia que nunca llega a superarse por completo" (p. 462). La distancia es inherente en la traducción, reiteramos que las diferencias idiomáticas se originan en las diferencias culturales. Es la traducción del puente entre dos culturas, donde la distancia se hace mínima y hasta puede desaparecer. El equilibrio del mundo social lo permiten las diversas lenguas.

"El problema hermenéutico no es pues un problema de correcto dominio de una lengua, sino del correcto acuerdo sobre un asunto, que tiene lugar en el medio del lenguaje" (p. 463), por lo cual, "asunto" se convierte en el núcleo de la traducción, al interpretar cada sociedad a través de lengua, pero sobre todo comprenderla. Este escenario quizá se pueda observar mejor en el caso de los bilingües. Hoy el número de bilingües ha crecido exponencialmente, entonces, ese acuerdo sobre el dominio del idioma se produce más y más en las mentes, individualmente de cada uno de estos bilingües. El cambio de una lengua a otra es automático, pero ¿será igual respecto a la cultura?

Gadamer continúa y afirma que "todo traductor es intérprete. El que algo esté en una lengua extraña no es sino un caso extremo de dificultad hermenéutica, esto es, de extrañeza y de superación de la misma" (p. 465). Aquí se centra el trabajo complementario del traductor -a veces no es suficiente un diccionario o base datos, o hasta el internet, en nuestros días-, el traductor se convierte en investigador, porque busca, indaga hasta escudriña (en el análisis etimológico, por ejemplo) el significado de la palabra a través de la interpretación, que debe superar la extrañeza del traductor en aras de la comprensión. Aquí se puede observar la experiencia del traductor, no solo en la praxis de la traducción sino también en la asimilación del espíritu de la palabra o texto. 
Se puede tener experiencia, pero es el espíritu que nos lleva a decir que la traducción también es arte. "Una traducción resulta tanto más lograda cuanta menos sensación se tiene de estar leyendo una traducción" (Grondin, 2008, p. 37). La hermenéutica para los traductores es comprender la naturaleza de la comprensión, es acercarse al texto en el idioma de origen de forma más honesta. El enfoque hermenéutico imprime coraje, porque en lo personal afirma la creatividad y no la necesidad de la traducción ideal, también evoca humildad porque comprensión del texto del idioma de origen siempre es relativo al propio texto original, ya que, el significado nunca puede imponerse o transponerse de una a otra. En cierto modo, el texto traducido, es un texto de segunda representación, porque solo el original es la fiel personalidad o intención del autor, entonces, para eliminar este estado de segundo orden con discurso transparente, el traductor debe lograr un texto traducido que puede tomarse como el original: La traducción es invisible, lo cual, por supuesto, solo se aprecia en el texto en LM.

\section{EL RETO DE LA TRADUCCIÓN}

El traductor debe estar familiarizado con la cultura, las costumbres y la configuración social de la fuente y el destino de los idiomas a traducir. También debe estar familiarizado con diferentes estilos de hablar y normas sociales (Yenkimaleki, 2016). Sin embargo, no implica que se logre la traducción ideal o deseada. Muchos son los retos del traductor, pero más son los retos de la traducción. Se observará en los siguientes acápites una compilación del desafío entre los textos y el traductor. Así, tratando de que las diferencias en el lenguaje que se ven a nivel superficial, bajo el cual, se supone, reside una base sólida de igualdad: lo que puede estar codificado de manera diferente, pero permanece igual en cualquier código (Horner \& Tetreault, 2016).

Una palabra o concepto puede connotar significados diferentes en otro idioma o puede estar ausente por completo, por lo que la relación entre los dos idiomas no es necesariamente simétrica. Dos palabras también pueden referirse al mismo objeto, y esto no necesariamente transmite el significado previsto del texto original. La idoneidad, fidelidad y lealtad al texto fuente puede resultar en un texto que no es fácilmente comprensible en la LM. Los supuestos implícitos del paradigma de la equivalencia suelen obligar a las personas a criticar una traducción porque determinadas palabras no han sido reemplazadas. Así, la famosa frase establecida: “Traduttore traditore" (Gambier, 2016, p. 889).

No estamos de acuerdo con el utilizar la dicotomía de fidelidad versus traición, para la traducción - intraducibilidad. Si bien aceptamos que la traducción fue y es aún realizada por aquel que considera que maneja dos idiomas y en el camino, efectivamente, traicionan, ya que no es solo conocer una lengua, tal como lo hemos explicado en capítulos anteriores, es mucho más. La traición en la traducción tiene una connotación negativa desde el principio mismo del rol del traductor. No hay lugar a la traición, solo a la imposibilidad, al rechazo, e intraducibilidad y por siempre, habrá equivocaciones, en este último caso, más en la interpretación (oral) que en la traducción (escrito). No existe la traducción perfecta, y si hubiera dicha "traición" ya se estaría considerando un problema ético, que lo tienen todas las profesiones u oficios. ¿Se podrán construir siempre las equivalencias entre las lenguas? La respuesta, veremos luego, es que es relativo. Tal como lo manifiesta 
Gadamer (1997, p. 464) "La exigencia de fidelidad que se plantea a una traducción no puede neutralizar la diferencia fundamental entre las lenguas." Revisemos, a nuestro criterio, los retos más importantes en la traducción:

a. La primera traducción: Es la traducción en base a las primeras palabras que el traductor identifique en la LM, podría decirse "lo primero que se le ocurra", porque es más natural, porque está enraizado en su competencia cognitiva del idioma, porque es su primer entendimiento o comprensión, entre otras. Entonces se busca la expresión idiomática y en algunos casos el famoso "no suena" o "no suena bien" en la LM, ayuda, aunque parezca inverosímil, a una mejor comprensión del valor sintagmático del texto de LO.

b. La polisemia: una palabra en un idioma determinado puede capturar múltiples sentidos relacionados. Tal polisemia puede trasladarse a otro idioma, de modo que una sola palabra en el idioma de destino también captura estos mismos sentidos múltiples relacionados. ¿Qué es lo que nos lleva a conceptuar de diferentes formas a una palabra o término? ¿La denotación de la palabra no cumple con la plasmación o manifestación del ser u objeto? Es propio del ser humano asimilar un fondo oculto de una experiencia, lo cual afecta indirectamente a las formas explícitas de conocimiento y conciencia, que se observa en el fenómeno de la polisemia. Los efectos de una experiencia pueden ser la base de la interpretación, con una participación y creación del uso de un término, pero con diferentes significados.

c. Las notas del traductor: nos hacen reflexionar en el modo en que se construyen las traducciones o como se realiza la traslación entre lenguas, y por supuesto, la subjetividad inherente al ser humano, por ende, al traductor. Existe un interés por estudiar estos paratextos de una traducción ya que pueden aportar datos valiosos respecto de las condiciones de producción y recepción del discurso traducido. Estos bordes del texto muchas veces presentan con mayor vigor la noción de traducción implícita en una cultura particular y en un contexto profesional. Hasta se considera que este paratexto permite la apertura de puertas de idiomas más permeables a la traducción (Spoturno, 2019). Consideramos que las notas a pie de página pueden ser terribles en cuanto a apariencia, no obstante, siempre y cuando estén para proporcionar información complementaria y establecer discrepancias con el texto original, está entendido y aceptado el uso de notas al pie, en síntesis, utilizados correctamente contribuyen a los juicios del contenido del texto traducido.

d. La intraducibilidad: Debemos admitir que la traducción, siempre incluirá segmentos de intraducibilidad, lo que conlleva a inevitables fracasos o pérdidas en la translación de lo que se dice en un idioma a otro, principalmente por campos semánticos diferentes, referencias intertextuales, diferencias sintácticas, modismos e incluso las connotaciones medio silenciosas, que alteran las definiciones del vocabulario original.

Ricoeur (2006) argumenta que la traducción encuentra resistencia en el sentido de que puede verse como una amenaza para la LM, ya que siempre podemos preguntarnos si esta lengua realmente puede decir lo que ya se dijo en la LO. Esta resistencia también puede provenir del otro lado, el de LO. Allí se expresa la presunción de intraducibilidad, 
es decir, que lo que se dice en un idioma no se puede decir en otro. La propia posición de Ricoeur es que se trata de una fantasía alimentada por la admisión banal de que el original no será duplicado por otro original” (p. 20), una fantasía que suele verse reforzada por otra fantasía, la de una traducción perfecta. El modo de existencia del ser humano mismo está esencialmente determinado por este estado de sumisión al lenguaje, que limita dramáticamente bastante su capacidad de autodeterminación. Entonces, ¿es positiva la intraducibilidad? ¿es natural? o ¿solo una barrera en la comprensión del mundo? o ¿es una barrera necesaria para la comprensión del mundo? Para Ricoeur los giros idiomáticos no transmiten los mismos legados culturales y si se añade la connotación, hace la tarea aún más difícil, por lo cual la resistencia a la traducción se ve reflejada en este contexto, la intraducibilidad se hace presente. Lo que puede desvirtuarse en el habla, fonéticamente, como broma, se presenta el siguiente enunciado en inglés: is it Chilli?, el cual también podría entenderse, como: is it Chile? Refiriéndose al país "Chile". "Chilli" se refiere a "chill” frío, por lo cual se estaría diciendo, “¿hace frío?” y si vamos al diccionario, encontraremos que "chilli" se refiere al "pimiento picante o ajp".

También se puede apreciar estos retos en base a los tipos de textos a traducir: en la traducción literaria, quizás sea la traducción más difícil de lograr, ya que el escenario es totalmente subjetivo: Desde la aparentemente frase sencilla: "Be or not to be, that is the question" de Hamlet (Shakespeare, 1998), cuya traducción también puede corresponder en su sencillez a "ser o no ser, esa es la cuestión", sin embargo, se puede ampliar y desarrollar toda una explosión de sentimientos, emociones, y hasta de filosofía. Esta frase puede sensibilizarnos de muchas formas, porque la interpretación esencial, no solo es la clásica interrogante de 'deber ser (o no ser)' sino cuál es el significado profundo que esconde en cada uno de nosotros, hasta el dilema de nuestra existencia como seres humanos. ¿Se puede utilizar en cualquier momento de la vida del hombre? Si bien la frase nace de la fatalidad y la duda, solo quien no ha tenido la experiencia de alguna de ellas, podría negar.

El traductor se convierte en la pluma liberada de las perspectivas epistémicas y, por lo tanto, cualquier habilidad, conocimiento o estrategia que puede ayudar al traductor a simpatizar con un autor puede ser de utilidad; peligroso, pero muchas veces inevitable. Hoy, por ejemplo, además de los estudios de traducción como profesión, se exige de un acervo en la especialidad de la traducción: para la traducción legal, ser traductor pero también abogado, para la traducción literaria, ser traductor y literato, y en este último escenario que se visualiza con mayor claridad la experiencia hermenéutica en la interpretación pues puede ser inducido por una amplia formación literaria como la crítica literaria, estilística, literatura comparada, estética, escritura creativa entre otras.

La traducción literaria es una de las más difíciles, a nuestro parecer, porque las palabras van cambiando, tienen giros y se acrecienta la necesidad de entender el mensaje, por ejemplo, traducir a Ricardo Palma resultaba difícil debido a la carga semántica y rasgos de las palabras. Así la palabra "recatado" que en la actualidad significa honesto, modesto, circunspecto o cauto, en aquellos tiempos significaba "pobre".

En la traducción técnico-científica, quizás sean los discursos que tienen menos problemas de traducción, pues plantean un texto de orden pragmático, no contienen 
oraciones exclamativas, desiderativas o imperativas y el léxico es esencialmente denotativo y no conmutable, pues ha sido creado para un uso específico con precisión técnica, información correcta y lenguaje actual. Sin embargo, cuanto más se mezcla la ciencia con la vida, tanto más ambigua resulta la expresión: Uno de los términos más comunes en estos tiempos de convulsión social es "stress", concebido como un término que se utiliza para denotar "estrés" como tensión física o muscular, por ejemplo. No obstante, su significado especializado es "esfuerzo": en Mecánica de Suelos (Ingeniería Civil) se utilizan dos clases de esfuerzos: (i) compresión-esfuerzo que tratan de comprimir el material y (ii) tracción-esfuerzo en sentido contrario al esfuerzo de compresión. No se debería utilizar la palabra "tensión" en este caso para describir este fenómeno, sin embargo, en la práctica, en las diferentes áreas de la ciencia y tecnología ya se ha generalizado la correspondencia stress-tensión y está a designar el estrés que ya muchos padecemos.

En la traducción jurídica, por la naturaleza de estos textos, que recogen las consecuencias de las decisiones políticas de las diversas culturas a través del tiempo, estos tienen un nivel de arcaísmo alto, por lo cual, son menos propensos a cambios en el tiempo. Esto parece contradictorio si observamos que la leyes o normas deben ir cambiando de acuerdo a la conducta o comportamiento de las personas que son el núcleo de toda sociedad, aunado a esto a los nuevos fenómenos sociales que influyen significativamente en ellos, por ejemplo, el caso de los delitos informáticos, que solo pudo haber sucedido gracias al desarrollo de la ciencia y la tecnología.

Una mención aparte es la traducción de textos filosóficos, quizás la más compleja. El filósofo suele crear su propio vocabulario, en que cada término está cargado de un significado especial que ha sido impreso por el filósofo y que integra su pensamiento total. La versión de estos términos y conceptos inéditos muchas veces crea escabrosos problemas de interpretación y traducción. Heidegger tiene particularidades en sus construcciones léxicas, como el "dasein", que ha sido traducido como "realidad de la verdad" y en forma literal podría ser "ser-ahî", traducción que también es considerada, y ampliando un poco quizás el pensamiento de Heidegger hasta se podría interpretar como realidad humana o existencia. Entonces un ser humano fáctico, que experimenta la vida, aquí, allá, en diferentes tiempos y culturas, y hasta la muerte se convierte en vida, porque también es una forma de existencia, ya que se conoce a la muerte sin haberlo experimentado como ser humano, es decir "haber muerto". Igualmente, tal como nosotros consideramos la traducción de "bedeutung" por "significado" mientras que también se puede considerar como "referencia"; "bild" por "figura" y también como "imagen"; lo mismo ocurriría con muchos otros términos y conceptos, lo que conlleva dificultades hermenéuticas.

La traducción se fundamenta en una labor de interpretación del texto: el traductor es lector del texto en LO y LM, navegando a través de la realidad del autor del original, que básicamente es la interpretación del original frente a su realidad. Lo maravilloso, tomando a Gadamer (1997), es que el horizonte del texto le permite interpretar con equilibrio, logrando inteligibilidad del texto en LO para todo el mundo, y coincidimos con Sandoval (2020) cuando sostiene que "la hermenéutica es una lógica de la interpretación y, por tanto, del sentido que pretende llegar a un conocimiento comprensible" (p. 17). 
Los retos en la traducción son infinitos, como lo es el lenguaje. El traductor tiene la tarea titánica de mantener reciprocidad entre el original y la traducción, que es primordialmente el "sentido", sin embargo, si se logra el "sentido y forma", se podría decir que se ha logrado una traducción ideal, aunque, el traductor siempre quedará endeudado sea por la imposición de su deber o por la supervivencia del texto ya que la composición de la semántica requiere la composición de la traducción. La razón es que hay expresiones ambiguas que deben recibir más de una traducción. Así pues, el proceso de traducción opera sobre árboles de análisis sintáctico que representan la derivación de las expresiones.

\section{CONCLUSIONES}

Consideramos que la hermenéutica como traducción se debe enfocar no solo en la comprensión del texto para poder traducirlo sino también en el significado de la interpretación y la compresión en el proceso de la traducción. Se está construyendo puentes entre culturas, lo que implica puentes entre diferentes observaciones de la realidad, por ende, podemos decir que el traductor se convierte en una etapa, dentro del proceso de traducción, en un investigador que debe realizar su estado del arte, ya que requiere de analizar y evaluar el mensaje para decodificarlo y trasladarlo a la LM, teniendo en cuenta el momento histórico, pero sobre todo las características de la LO en el que fue codificado. La comprensión de textos se constituye en el pilar del traductor.

La traducción se convierte entonces en arte y ciencia, conjugadas para el uso de un conjunto de códigos adoptados por las distintas sociedades y culturas: El idioma que expresa la esencia universal e inherente del ser humano plasmado en el lenguaje. La traducción es esa relación biunívoca que tenemos con la vida, realidad, y con el mundo.

\section{Nota}

1 El presente manuscrito derivado de la investigación "la perspectiva hermenéutica como comprensión de la denotación del lenguaje”, como requisito para la obtención del grado de doctor en filosofía por la Universidad Nacional Mayor de San Marcos.

\section{ORCID}

Teresa Jesús Ríos Delgado: Universidad Nacional Mayor de San Marcos, Lima, Perú

\section{FUENTE DE FINANCIAMIENTO}

El estudio fue autofinanciado.

\section{CONFLICTOS DE INTERÉS}

El autor declara que no existe conflicto de interés.

\section{AGRADECIMIENTO}

No aplica.

\section{PROCESO DE REVISIÓN}

Este estudio ha sido revisado por pares externos en modalidad de doble ciego. 


\section{DECLARACIÓN DE DISPONIBILIDAD DE DATOS}

No aplica.

\section{REFERENCIAS BIBLIOGRÁFICAS}

Gadamer, H. G. (1997). Verdad y Método. Fundamentos de una hermenéutica filosófica. Salamanca: Ediciones Sigueme.

Gambier, Y. (2016). Rapid and Radical Changes in Translation and Translation Studies. International Journal of Communication 10(10), 887-906.

Grondin, J. (1999). Introducción a la hermenéutica filosófica. Barcelona: Herder.

Grondin, J. (2008). ¿Qué es la Hermenéutica? Barcelona: Herder.

Horner, B., \& Tetreault, L. (2016). Translation as (Global) Writing. Composition Studies, 44(1), 13-30.

Mansoor, K. (2018). Translation Across the Difficulties of Equivalence Concept. Transactions on Modern Languages, 17(1), 55-66.

Quintana, L., \& Hermida, J. (2019). La hermenéutica como método de interpretación de textos en la investigación psicoanalítica. Perspectivas en psicología, 16(2), 73-80.

Ricoeur, P. (2006). On Translation. New York: Routledge.

Ricoeur, P. (2006). Teoría de la Interpretación. Discurso y excedente de sentido (6 ed.). Madrid: Siglo XXI Editores.

Sandoval, E. (2020). Hermenéutica: tensiones, condiciones y abismos en la interpretación. Andamios, 17(43), 13-29. doi:10.29092/uacm. v17i43.762

Sang, Z. (2018). How does the context make a translation happen? An activity theory perspective. SOCIAL SEMIOTICS, 28(1), 125-141. doi:10.1080/10350330.2017.1 336848

Shakespeare, W. (1998). Hamlet, Prince of Denmark. Collins edition.

Spoturno, M. (2019). La conquista del espacio enunciativo. Un estudio de las notas en la traducción al español de Borderlands/La Frontera. LENGUA Y HABLA (23), 360379 .

Trăuşan-Matu, Ş. (2017). Hermenophore tools, a new perspective on text analysis. Revista Romana de Interactiune Om-Calculator, 10(1), 75-88.

Tutone, M. (2019). El traductor de Gadda, un filólogo equilibrista. Hermēneus. Revista de traducción e interpretación, 21, 355-386. doi:10.24197/her.21.2019.355-386

Wittgenstein, L. (1988). Investigaciones Filosóficas. (A. García Suárez, \& U. Moulines, Trans.) Barcelona, España: Grijaldo.

Yenkimaleki, M. (2016). Stepping into others' shoes: the readership taste in translation. Journal of Linguistic Intercultural Education, 9(1), 139-149.

Rios Delgado, T. J. (2022). La hermenéutica y los textos: el reto de la traducción. Puriq, 4, e217. https://doi.org/10.37073/puriq.4.1.217 